\title{
Classical and Thermodynamic Stability of Black Branes
}

\author{
Harvey S. Reall \\ Physics Department, Queen Mary College \\ Mile End Road, London E1 4NS, United Kingdom \\ 8 April 2001 \\ Preprint QMUL-PH-01-06
}

\begin{abstract}
It is argued that many non-extremal black branes exhibit a classical Gregory-Laflamme instability if, and only if, they are locally thermodynamically unstable. For some black branes, the Gregory-Laflamme instability must therefore disappear near extremality. For the black $p$-branes of the type II supergravity theories, the Gregory-Laflamme instability disappears near extremality for $p=1,2,4$ but persists all the way down to extremality for $p=5,6$ (the black D3-brane is not covered by the analysis of this paper). This implies that the instability also vanishes for the near-extremal black M2 and M5-brane solutions.
\end{abstract}

\section{Introduction}

\subsection{The Gregory-Laflamme instability}

The Gregory-Laflamme (GL) instability is a classical instability of non-extremal black branes [1, 2]. The existence of this instability is often explained by arguing that a black $p$-brane has a lower entropy per unit $p$-volume than an array of black holes with the same total mass and charge per unit volume円, and must therefore be unstable. However, this explanation leaves something to be desired because it uses thermodynamic (and therefore quantum) arguments to explain a classical phenomenon.

It is, of course, well-known that the classical laws of black hole mechanics [3] can be reinterpreted as thermodynamic laws once it is appreciated that black holes emit thermal radiation [4] and therefore have a temperature and associated entropy. Conversely, the laws of thermodynamics applied to black holes can sometimes be reformulated in classical language. The entropy argument above would become an argument based on the second law of black hole mechanics [5], which states that the area of the event horizon is non-decreasing in any physical process. Since the area of the horizon would increase if a black brane were to evolve to an array

\footnotetext{
1 Assuming that the black holes can carry the charge of the black brane. If not, then one might instead consider an array of uncharged black holes threaded by an extremal black brane.
} 
of black holes, the second law certainly permits a classical instability of black branes. However, it does not require it.

\subsection{The Gubser-Mitra conjecture}

The GL instability has recently been re-examined by Gubser and Mitra (GM) [6, 0] from the perspective of the AdS/CFT correspondence [8]. It was argued that the absence of tachyonic modes in the AdS glueball calculations [9, 10] is evidence that near-extremal D3-branes are in fact classically stable, and that this is consistent with the work of GL because GL only checked that an instability exists for branes that are far from extremality. However, this appears to contradicts the claim of GL [1] that their instability persists all the way down to extremality 2 . Furthermore, to apply AdS/CFT arguments one must first take the decoupling limit [8] of the black brane, which makes it only infinitesimally non-extremal[?

More interestingly, the following conjecture was made by GM: a black brane with a noncompact translational symmetry is classically stable if, and only if, it is locally thermodynamically stable. The definition of local thermodynamic stability adopted by GM was the one used in [12], namely that the Hessian of the entropy with respect to the other extensive thermodynamic variables (i.e. mass and charges) should be negative definite. This is the mathematical statement of the condition that it shouldn't be possible for the system to gain entropy by splitting into several parts whilst conserving energy and charge, i.e., it is a statement about the microcanonical ensemble.

At first sight, the GM conjecture appears to contradict the claim [11] that the GL instability persists all the way down to extremality. For example, near-extremal D3-branes have positive specific heat, which implies that they satisfy the above definition of local thermodynamic stability and should therefore be classically stable according to the conjecture. However, the work of GL only covered black branes carrying magnetic charge with respect to a Neveu-Schwarz $n$-form. Therefore, the only black brane of type II supergravity covered by their work is the NS5-brane. This brane does indeed have negative specific heat all the way down to extremality, so the fact that it is classically unstable agrees with the GM conjecture. One might think that the work of GL could be extended to the F-string by dualizing the NS 3-form, but this changes the coupling to the dilaton, taking one outside the class of theories considered by GL. One of the results of the present paper will be to show that the GL instability occurs for many more solutions than just the ones considered by GL.

The condition that there should be some non-compact translational symmetry was included in the GM conjecture to exclude black holes from the scope of the conjecture. This is because it is well-known that many black holes, for example Schwarzschild, are classically stable [13] yet have negative specific heat and are therefore locally thermodynamically unstable.

The GM conjecture can be regarded as a refinement of the entropy argument mentioned in the first paragraph of this paper. This entropy argument refers to global thermodynamic stability. It seems much more plausible that, if there is a connection between classical and

${ }^{2}$ That is, for any non-zero value of the non-extremality parameter. It was argued that the instability is absent for extremal branes, which is as one would expect in a supersymmetric theory.

${ }^{3}$ Thanks to Simon Ross for emphasizing this point to me. Throughout this paper, "near-extremal" will mean that some non-extremality parameter lies in a finite neighbourhood of its extremal value. 
thermodynamic stability, it should be local thermodynamic stability that is relevant. For example, one might imagine some kind of potential barrier separating the black brane from an array of black holes, so the only way that the brane could gain in entropy would be by quantum tunneling through this barrier. In that case, since the instability would be quantum mechanical in nature, global thermodynamic instability would not imply classical instability.

The evidence that GM gave for their conjecture was based on studying black holes solutions [14] of gauged $\mathcal{N}=8$ supergravity in four dimensions. These solutions are asymptotic to anti-de Sitter space and the black holes considered were large compared with the AdS radius of curvature. This presumably implies that the curvature of their horizons should not be significant so they would be expected to exhibit similar behaviour to black branes. Therefore if the conjecture is correct, then it should apply to these black holes too. It was found that a classical instability appeared almost precisely where local thermodynamic stability was lost, with a small discrepancy arising from numerical error. Note that large asymptotically flat black holes would not be expected to behave like black branes because there is no length scale with which the curvature of the horizon can be compared.

\subsection{Proving the conjecture}

In this paper, a semi-classical proof of the GM conjecture will be given, using the Euclidean path integral approach to quantum gravity. This has been succesful in providing a semi-classical explanation of other connections between classical and thermodynamic properties of black holes, for example the relationship between the entropy of a black hole and the area of its horizon [15]. It is convenient to review some basic facts about this approach [16]. The Euclidean path integral for the canonical ensemble is

$$
Z=\int d[\mathbf{g}] \exp (-I[\mathbf{g}])
$$

where $I$ denotes the Euclidean Einstein-Hilbert action. The path integral is taken over Riemannian manifolds $(M, \mathbf{g})$ that are asymptotically flat, i.e., tend to the flat metric on $S^{1} \times R^{3}$ outside some compact region. The $S^{1}$ should have proper length $\beta=1 / T$, where $T$ is the temperature.

This path-integral is only well-defined in the semi-classical approximation. In this approximation, one looks for saddle-points of the Euclidean action, i.e., solutions of the Euclidean Einstein equations. These are referred to as gravitational instantons. Near a gravitational instanton, the metric can be written as

$$
g_{\mu \nu}=\bar{g}_{\mu \nu}+\delta g_{\mu \nu},
$$

where $\bar{g}_{\mu \nu}$ is the metric of the instanton, and $\delta g_{\mu \nu}$ denotes the perturbation. One can add gauge fixing and ghost terms to the action to deal with the gauge freedom in $\delta g_{\mu \nu}$. The action can be expanded around the saddle point:

$$
I[\mathbf{g}]=I_{0}[\overline{\mathbf{g}}]+I_{2}[\overline{\mathbf{g}}, \delta \mathbf{g}]
$$

where $I_{2}$ is quadratic in the fluctuation. The contribution to $I_{2}$ from the trace of the metric perturbation is a wrong-sign kinetic term. This is the well-known conformal factor problem [17]. 
Nothing new will be said about this problem in the present paper. The part of $I_{2}$ involving the traceless part of the metric perturbation is, in transverse gauge, proportional to

$$
\int d^{4} x \sqrt{\bar{g}} H^{\mu \nu} \Delta_{L} H_{\mu \nu}
$$

where $H_{\mu \nu}$ is transverse traceless and $\Delta_{L}$ is the Euclidean Lichnerowicz operator. The pathintegral over the transverse traceless part of the metric perturbation can be evaluated by expanding $H_{\mu \nu}$ in eigenfunctions of $\Delta_{L}$, i.e., solutions of

$$
\Delta_{L} H_{\mu \nu}=\lambda H_{\mu \nu}
$$

The boundary conditions are that $H_{\mu \nu}$ should vanish fast enough at infinity to have finite norm, and be regular everywhere else. The contribution to the partition function from the instanton is

$$
A \exp \left(-I_{0}[\overline{\mathbf{g}}]\right)
$$

where $A$ is a functional determinant that arises from the path-integrals over the metric perturbation and ghosts. In particular, it includes a factor of $\left(\operatorname{det} \Delta_{L}\right)^{-1 / 2}$. This implies that if $\Delta_{L}$ has a negative eigenvalue then there will be an imaginary contribution to the path integral. This pathology arises from the fact that the canonical ensemble for gravity is not well-defined in infinite volume [18, 16, i.e., it is an indication of a thermodynamic instability.

For the Euclidean Schwarzschild solution, there is a single transverse traceless negative mode [19, 20, i.e., a solution to equation 1.5 with negative $\lambda$. The eigenvalue is 20]

$$
\lambda=-0.19 M^{-2}
$$

where $M$ denotes the Schwarzschild mass. The existence of this negative mode implies that the Euclidean Schwarzschild solution is merely a saddle point of the Euclidean action, rather than a local minimum [19. Therefore this instanton cannot be regarded as giving an approximation to the partition function. The correct interpretation is as an instanton describing a non-perturbative instability of flat space at finite temperature [20].

It is now possible to explain the main idea of this paper. Consider an uncharged black $p$-brane in $4+p$ dimensions with metric

$$
d s^{2}=g_{\mu \nu} d x^{\mu} d x^{\nu}+\delta_{i j} d z^{i} d z^{j}
$$

where $g_{\mu \nu}$ denotes the four dimensional Lorentzian Schwarzschild metric and $z^{i}$ denote the flat spatial worldvolume directions of the brane. GL considered [1] metric perturbations of this solution of the form

$$
h_{\mu \nu}=\exp \left(i \mu_{i} z^{i}\right) H_{\mu \nu}, \quad h_{\mu i}=h_{i j}=0,
$$

where transverse traceless gauge is used for $h_{\mu \nu}$, and $H_{\mu \nu}$ is spherically symmetric. GL looked for modes that are regular on the future horizon of the brane and decay at infinity, but grow exponentially in time: $H_{\mu \nu} \propto \exp (\Omega t)$. The equation of motion for the perturbation is [回]

$$
\tilde{\Delta}_{L} H_{\mu \nu}=-\mu^{2} H_{\mu \nu}
$$

where $\tilde{\Delta}_{L}$ denotes the four dimensional Lorentzian Lichnerowicz operator and $\mu^{2}=\sum_{i} \mu_{i} \mu_{i}$. 
GL showed that there is a critical value $\mu_{*}$ such that for every $\mu<\mu_{*}$, equation 1.10 has a solution that is regular on the future horizon and decays at spatial infinity, but grows exponentially in time. For $\mu>\mu_{*}$, no growing mode was found and the solutions are oscillatory in time. It follows that the solution with $\mu=\mu_{*}$ must be independent of time. This solution will be referred to as the threshold unstable mode. Note that the classical second law implies that a black brane compactified on a sufficiently small torus cannot evolve to an array of black holes, which suggests that the black brane should be stable against small wavelength (large $\mu$ ) perturbations, and therefore that a time-independent mode must exist if the brane is unstable to large wavelength perturbations. So one can argue that the existence of such a mode is necessary for a classical instability without referring to the numerical results of [1, 2]. This will be important for the more general black brane solutions considered later in this paper.

It is now possible to make contact with the Euclidean path integral by Wick rotating $H_{\mu \nu}$ for the threshold unstable mode. Since $H_{\mu \nu}$ is static and spherically symmetric, Wick rotation simply changes the sign of $H_{t t} . H_{\mu \nu}$ is translationally invariant on the brane world-volume so it can be dimensionally reduced to four dimensions. Wick rotation transforms $\tilde{\Delta}_{L}$ into $\Delta_{L}$, so equation 1.10 turns into equation 1.5 with $\lambda=-\mu_{*}^{2}$. Hence it appears that the classical Lorentzian threshold unstable mode corresponds precisely to a Euclidean negative mode. It will be shown later that the Wick rotated mode does indeed satisfy the correct boundary conditions for a negative mode.

This argument can be checked by comparing the numerical results of [20] and [1]. The latter gave results for horizon radius $r_{+}=2$ [2], which corresponds to $M=1$, i.e., $\lambda=-0.19$. This would give $\mu_{*}=0.44$, which is in excellent agreement with figure 1 of [1]. It is possible to extend this matching to the other dimensions for which the negative mode has been calculated [21], namely $\lambda=-1.60 r_{+}^{-2}$ in five dimensions and $\lambda=-3.39 r_{+}^{-2}$ in seven dimensions. For $r_{+}=2$, this gives $\mu_{*}=0.63$ and $\mu_{*}=0.92$ respectively, again in good agreement with [四]. A simple scaling argument shows that this agreement must extend to all values of $r_{+}$.

It is clear that the classical GL instability of the black brane is very closely related to the thermodynamic instability of the Schwarzschild black hole. The threshold unstable mode is related to the Schwarzschild negative mode by discarding the $\exp \left(i \mu_{i} z^{i}\right)$ factor and Wick rotating . The existence of negative modes is closely linked to local thermodynamic stability, as will be explained shortly. It therefore seems likely that the above argument can be generalized into a proof of the GM conjecture for more general black brane solutions. That will be the subject of the rest of this paper.

\subsection{Outline of this paper}

The next section describes the class of black branes that will be discussed in this paper, namely magnetically charged dilatonic black branes. In section 3, the relationship between local thermodynamic stability and negative modes is explained. Section 1 discusses classical perturbations and in section 5 it is shown how these are related to negative modes. The final section summarizes the main conclusions and suggests directions for future research.

\footnotetext{
4 This argument shows how a negative mode of the Schwarzschild black hole is related to a static zero mode of the black string. This relationship was noted in [22], although the connection with classical stability was not appreciated (indeed it was argued that the black string is classically stable!)
} 


\section{Black brane solutions}

This paper will discuss black $p$-brane solutions of the action

$$
I=\epsilon \int d^{D} x \sqrt{\epsilon g}\left(-R+\frac{1}{2}(\partial \phi)^{2}+\frac{1}{2 n !} e^{a \phi} F_{(n)}^{2}\right),
$$

where $\epsilon=+1$ for Euclidean signature and -1 for Lorentzian signature. $F_{(n)}$ denotes a $n$-form field strength obtained from a $(n-1)$-form potential $A_{(n-1)}$ by $F_{(n)}=d A_{(n-1)}$.

Black brane solutions of this action have been given by several authors [23, 24, 25, 26]. The notation of [26] will be used here. The magnetically charged black brane solutions can be written:

$$
\begin{aligned}
d s^{2} & =\left(1+\frac{k}{r^{\tilde{d}}} \sinh ^{2} \mu\right)^{-\frac{4 \tilde{d}}{\Delta(D-2)}}\left(\epsilon e^{2 f} d t^{2}+d z^{i} d z^{i}\right) \\
& +\left(1+\frac{k}{r^{\tilde{d}}} \sinh ^{2} \mu\right)^{-\frac{4 d}{\Delta(D-2)}}\left(e^{-2 f} d r^{2}+r^{2} d \Omega_{\tilde{d}+1}^{2}\right), \\
e^{-\frac{\Delta}{2 a} \phi} & =1+\frac{k}{r^{\tilde{d}}} \sinh ^{2} \mu, \quad e^{2 f}=1-\frac{k}{r^{\tilde{d}}} .
\end{aligned}
$$

The parameters $d, \tilde{d}$ and $\Delta$ are defined by

$$
\tilde{d}=n-1, \quad d=p+1=D-\tilde{d}-2, \quad \Delta=a^{2}+\frac{2 d \tilde{d}}{D-2} .
$$

The $n$-form field strength is a constant times the volume form on $S^{n}$. The two free parameters $k$ and $\mu$ are related to the charge $\lambda$ and mass per unit $p$-volume $m$ by [26]

$$
\lambda=\frac{\tilde{d} k}{\sqrt{\Delta}} \sinh 2 \mu, \quad m=k\left(\frac{4 \tilde{d}}{\Delta} \sinh ^{2} \mu+\tilde{d}+1\right) .
$$

The temperature is

$$
T=\frac{\tilde{d}}{4 \pi r_{+}}(\cosh \mu)^{-4 / \Delta} .
$$

The extremal limit is $k \rightarrow 0, \mu \rightarrow \infty$ with $k e^{2 \mu}$ fixed. It is straightforward to verify that the specific heat at fixed charge is always negative if $2 \tilde{d} / \Delta \leq 1$. However, if $2 \tilde{d} / \Delta>1$ then this specific heat is negative far from extremality but positive near extremality.

For the black $p$-branes of the type II supergravity theories [23], with the exception of the D3-brane, one can always dualize to make the solution magnetically charged. The solutions then take the above form, and one finds that the specific heat changes sign near extremality when $p \leq 4$ (i.e., D0, F1, D1, D2, D4) but not for $p=5,6$ (NS5, D5, D6) (D p-branes with $p>6$ will not be considered here because they are not asymptotically flat). For the solutions considered by Gregory and Laflamme, the specific heat never changes sign. The specific heat of the D3-brane does change sign but this brane lies outside the class of solutions considered in this paper. Nevertheless, it seems likely that the arguments of the present paper could be generalized to include this case. For the black M2 and M5 brane solutions [24] of eleven 
dimensional supergravity, the specific heat is negative far from extremality but becomes positive near extremality.

Dimensional reduction on the worldvolume of the above solution leads to a magnetically charged dilatonic black hole solution in $n+2$ dimensions. The properties of such solutions were extensively discussed in [27]. It is clear that the temperature of this black hole must be the same as the temperature of the black brane. It is also the case that the charge of the black hole is the same as the charge of the black brane, and the mass of the black hole is proportional to the mass per unit $p$-volume of the black brane [28]. It follows that the specific heat at constant charge of the black hole has the same sign as the specific heat at constant charge of the black brane.

\section{$3 \quad$ Negative modes and the specific heat}

\subsection{Canonical ensemble}

The canonical ensemble for gravity can be rendered well-defined by imposing boundary conditions at a finite boundary rather than at infinity [29]. Consider a spherical cavity of surface area $4 \pi r_{B}^{2}$, whose boundary is held at a fixed temperature $T$. The partition function in the canonical ensemble is given by a path integral over Riemannian geometries $(M, \mathbf{g})$ such that $\partial M=S^{1} \times S^{2}$, where the $S^{1}$ has proper length $\beta=1 / T$ and the $S^{2}$ has area $4 \pi r_{B}^{2}$.

In the case of a pure gravity theory, for small $\operatorname{Tr}_{B}$ the only solution to the Euclidean Einstein equations that obeys these boundary conditions is flat space with a periodically identified time coordinate. For larger $\operatorname{Tr}_{B}$, there are three solutions: flat space, a small Schwarzschild black hole, and a large Schwarzschild black hole (with size of order $r_{B}$ ). The smaller black hole has negative specific heat and the larger black hole has positive specific heat.

It is straightforward to demonstrate that the black hole with negative specific heat cannot be a local minimum of the Euclidean action and must therefore have a negative mode. For a Schwarzschild black hole, this was shown in [30] using a construction given in [31]. The idea is to construct a 1 parameter family of geometries for which the Euclidean action (relative to flat space) takes the form

$$
I=\beta E(x)-S(x),
$$

where $x$ is the parameter labelling the path. There should be should be some value $x=x(T)$ for which the geometry becomes the black hole geometry of interest. At this value, $E$ and $S$ are the energy and entropy of the black hole:

$$
E(x(T))=E(T), \quad S(x(T))=S(T) .
$$

For other values of $x$, the interpretation of the functions $E$ and $S$ is not important. The black hole extremizes the action, so at $x=x(T)$,

$$
\left(\frac{\partial I}{\partial x}\right)_{T}=0
$$

which is simply the condition for thermal equilibrium of the black hole with a heat bath of temperature $T$ :

$$
\left[\beta \frac{d E}{d x}-\frac{d S}{d x}\right]_{x=x(T)}=0 \Rightarrow\left[\frac{d E}{d S}\right]_{x=x(T)}=T \text {. }
$$


The second derivative of the action along the path parametrized by $x$ is

$$
\left(\frac{\partial^{2} I}{\partial x^{2}}\right)_{T}=\left(T \frac{d x}{d T}\right)^{-2} \frac{d E}{d T} .
$$

It is evident from this equation that if the specific heat $d E / d T$ is negative then the black hole cannot be a local minimum of the action and therefore it must have a negative mode?.

In order to exhibit this connection between specific heat and negative modes it was necessary to assume that there exists a 1-parameter family of geometries, including the black hole, for which equation 3.1 is obeyed. This will now be demonstrated for the black holes of relevance to this paper, namely those obtained by dimensional reduction of the black brane solutions given above. These are dilatonic black holes in $n+2$ dimensions carrying magnetic charge with respect to a $n$-form field strength. The construction is a straightforward generalization of 31]. The Euclidean metric can be written

$$
d s^{2}=U(r) d \tau^{2}+V(r)^{-1} d r^{2}+R(r)^{2} d \Omega_{n}^{2} .
$$

The exact form of $U(r), V(r)$ and $R(r)$ will not be important. The dilaton is a function of $r$ alone, and the $n$-form is proportional to the volume form on $S^{n}$.

In the canonical ensemble, if the boundary is at $r=r_{B}$ then the proper length of the Euclidean time direction at $r=r_{B}$ should be $\beta=1 / T$. It is convenient to normalize $\tau$ so that $\tau \sim \tau+\beta$, which implies $U\left(r_{B}\right)=1$. The functions $U(r)$ and $V(r)$ both vanish at the horizon, $r=r_{+}$. The condition for regularity at $r=r_{+}$is

$$
\sqrt{U^{\prime}\left(r_{+}\right) V^{\prime}\left(r_{+}\right)}=\frac{4 \pi}{\beta},
$$

which gives $r_{+}=r_{+}(T)$. The black hole solution is specified by the parameters $k$ and $\mu$, which are determined by the temperature $T$ and charge $\lambda$. This black hole will be referred to as $B(T, \lambda)$.

The other geometries in the family will be taken to have a metric of the form 3.6 but now with $U(r)$ treated as an arbitrary function. $V(r), R(r)$, the dilaton and the $n$-form are all taken to be the same as for a black hole with charge $\lambda$ but arbitrary temperature. In other words, $k$ and $\mu$ are constrained only to keep $\lambda$ fixed but not $T$, so this gives a 1-parameter family of metrics on the surfaces of constant $\tau$. This family can be parametrized by the value of $r_{+}$. Choosing $r_{+}=r_{+}(T)$ reproduces the geometry of the spatial sections of $B(T, \lambda)$.

This choice of geometry for surfaces of constant $\tau$ implies that the constraint equations of general relativity are satisfied on these surfaces. $\tau$ is still taken to be identified with period $\beta$. The only restrictions on $U(r)$ are $U\left(r_{B}\right)=1$ (so the metric obeys the boundary conditions), $U\left(r_{+}\right)=0$ (so the topology is the same as that of the black hole $B(T, \lambda)$ ), that $U^{\prime}\left(r_{+}\right)$obeys equation 3.7 (so that the metric is regular at $r=r_{+}$) and that $U(r)$ is positive for $r>r_{+}$. It is obviously possible to choose a suitable family of functions $U(r)$ so that the metric becomes that of $B(T, \lambda)$ when $r_{+}=r_{+}(T)$.

\footnotetext{
${ }^{5}$ Note that the path of off-shell geometries parametrized by $x$ does not correspond directly to an eigenfunction of the Lichnerowicz operator, but rather to some linear combination of eigenfunctions. Since the action decreases along this path, at least one of these eigenfunctions must have a negative eigenvalue.
} 
The action of these geometries (relative to flat space) can be calculated using the methods of [16, 32]. One first excises the region $r<r_{+}+\epsilon$. In the region $r>r_{+}+\epsilon$, one write the action in Hamiltonian form. The contribution from this region is then simply

$$
I_{+}=\beta H,
$$

where $H$ is the Hamiltonian evaluated on the above metric. Since the above metric is static and satisfies the constraint equations, the only contribution to $H$ comes from a surface term at $r=r_{B}$, giving

$$
I_{+}=\beta E,
$$

where $E$ depends only on $r_{+}$and the charge $\lambda$. The total action is given by adding the action of the region $r<r_{+}+\epsilon$. In the limit $\epsilon \rightarrow 0$, this is given entirely by a gravitational surface term国 at $r=r_{+}$which, after using equation 3.7 reduces to

$$
I_{-}=-\frac{A}{4 G},
$$

where $A$ is the area of the horizon at $r=r_{+}$, and $G$ is Newton's constant. The total action is therefore

$$
I=I_{+}+I_{-}=\beta E-S,
$$

where $S=A / 4 G$, which depends only on $r_{+}$. This establishes equation 3.1 (with $x=r_{+}$). The proof that negative specific heat implies the existence of a negative mode then proceeds as above. The only subtlety is that, since the charge was held fixed above, the specific heat should be calculated at constant charge. The reason for working in the canonical ensemble (fixed charge) rather than the grand canonical ensemble (fixed potential) is that the theory does not contain charged particles and therefore the charge cannot fluctuate. This is also true for electrically charged black holes].

\subsection{Physical interpretation}

There is a simple physical interpretation of the off-shell geometries considered above. Consider a typical member of this family. The geometry of the spatial sections is simply that of a black hole of charge $\lambda$ but with temperature $T^{\prime} \neq T$. Since the metric is static (independent of $\tau$ ) analytic continuation to Lorentzian signature at a surface of constant $\tau$ yields initial data for solving the Lorentzian Einstein equations and the unique solution is the black hole with charge $\lambda$ and temperature $T^{\prime}$. In other words, the path of off-shell geometries constructed above corresponds to an instability in which the black hole changes temperature at fixed charge, which is precisely the instability that one would expect for a black hole in contact with a heat bath. Note that the off-shell geometry is not simply given by analytic continuation of the metric of a black hole of temperature $T^{\prime}$ since this would not give a metric with the correct period for the Euclidean time at $r=r_{B}$. Note also, that if this interpretation is correct then many different $U(r)$ correspond to the same Lorentzian black hole.

\footnotetext{
${ }^{6}$ A surface term involving the $n$-form vanishes for magnetic black holes.

${ }^{7}$ In the case of electric charge (for $n=2$ ), it is necessary to add an extra surface term to the Euclidean action when working in the canonical ensemble [33, 34].
} 
It has been shown that negative specific heat implies the existence of a negative mode. To see that this is a physical negative mode (rather than a negative mode arising from the conformal factor problem), consider the off-shell geometries near $B(T, \lambda)$. These can be regarded as perturbations of $B(T, \lambda)$. The function $U(r)$ is essentially arbitrary for $r_{+}<r<r_{B}$, so by adjusting $U(r)$, the trace of the metric perturbation can be freely adjusted. If the negative mode were associated with the conformal factor problem then one would expect increasing the trace of the metric perturbation to make the action more negative. However, since the constraint equations are satisfied on surfaces of constant $\tau$, the action does not depend on $U(r)$ and therefore this change in the trace of the metric perturbation does not change the action. It follows that the negative mode whose existence was demonstrated above is a non-conformal negative mode.

The above discussion considered black holes in finite cavities. This is a rather unphysical situation since it is hard to see how an appropriate cavity could be constructed. The rest of this paper will consider only black holes in infinite space, i.e., the $r_{B} \rightarrow \infty$ limit will be assumed.

\subsection{Other ensembles}

Since the charge of the black hole cannot fluctuate, the condition for local thermodynamic stability in the microcanonical ensemble is that the second derivative of the entropy with respect to the mass must be negative. Equivalently, the specific heat should be positive. So the condition for local thermodynamic stability of the black hole is the same in the canonical and microcanonical ensembles.

In a theory containing charged particles, one might choose to use the grand ensemble for electrically charged holes. This could lead to new negative modes involving the gauge field. For example, consider the Reissner-Nordstrom solution. The necessary and sufficient conditions for local thermodynamic stability in the grand ensemble is that the Hessian of the Gibbs free energy with respect to the entropy $S$ and charge $Q$ be positive definite. This is equivalent to

$$
\left(\frac{\partial E}{\partial T}\right)_{Q}>0, \quad\left(\frac{\partial \Phi}{\partial Q}\right)_{T}>0,
$$

where $\Phi$ denotes the potential at infinity (or at $r=r_{B}$ ) in a gauge regular at the horizon. In four dimensions, one finds that the former equation is violated for $Q / M<\sqrt{3} / 2$ but the latter equation is violated for $Q / M>\sqrt{3} / 2$. Thus one would expect an instability analagous to the thermodynamic instability of the Schwarzschild black hole for small $Q$, and the associated negative mode would involve the metric as above. However, for large $Q$, the instability involves the black hole gaining or losing charge, so the instability would involve the electromagnetic field and charged matter fields, as for the black holes studied in [6, 7]. Hence, in this case one would expect a negative mode involving the electromagnetic and matter fields. In a theory that did not contain charged matter, one would work in the canonical ensemble and the second of the above stability conditions would not be required so one would expect no negative mode for $Q / M>\sqrt{3} / 2$. This is confirmed by numerical calculations [36], which show that the negative mode involving the metric vanishes precisely at $Q / M=\sqrt{3} / 2$.

\footnotetext{
${ }^{8}$ See section 21 of [35] and replace $-V$ with $Q$ and $p$ with $\Phi$.
} 
Note that it has only been shown that negative specific heat implies the existence of a negative mode. Conversely, the existence of a negative mode implies that the black hole is only a saddle point of the Euclidean action, which implies that it suffers from some pathology in the canonical ensemble. This suggests that it should be locally thermodynamically unstable. The Reissner-Nordstrom example is evidence that this is true, but I am unaware of a more general proof.

\section{Classical stability}

The aim of this section is to derive the equations of motion for classical perturbations of the black brane solutions discussed in section 2 .

\subsection{Equations of motion}

The analysis will be simplified by a convenient choice of conformal frame. For the moment the choice of conformal frame will be kept arbitrary by writing

$$
\hat{g}_{M N}=e^{2 b \phi} g_{M N}
$$

where a caret refers to the $D$-dimensional Einstein metric. In the new frame, the action is

$$
I=\epsilon \int d^{D} x \sqrt{\epsilon g}\left[e^{-\beta \phi}\left(-R+k(\partial \phi)^{2}\right)+\frac{1}{2 n !} e^{\alpha \phi} F_{(n)}^{2}\right]
$$

where

$$
\beta=-(D-2) b, \quad k=\frac{1}{2}-(D-1)(D-2) b^{2}, \quad \alpha=a+(D-2 n) b .
$$

The equations of motion can be written

$$
\begin{gathered}
\nabla_{M}\left(e^{\alpha \phi} F^{M N_{1} \ldots N_{n-1}}\right)=0 \\
\nabla^{2} \phi-\beta(\partial \phi)^{2}=\frac{a}{2 n !} e^{(\alpha+\beta) \phi} F^{2} \\
R_{M N}=\left(k+\beta^{2}\right) \partial_{M} \partial_{N} \phi-\beta \nabla_{M} \nabla_{N} \phi \\
+\frac{1}{2(n-1) !} e^{(\alpha+\beta) \phi} F_{M P_{1} \ldots P_{n-1}} F_{N} P_{1} \ldots P_{n-1}-\frac{\Lambda}{4 n !} e^{(\alpha+\beta) \phi} F^{2} g_{M N},
\end{gathered}
$$

where

$$
\Lambda=\left(\frac{D-2 n}{D-2}\right)\left(\frac{2 \beta^{2}}{D-2}-1\right)+\frac{2 \alpha \beta}{D-2}+1
$$

The conformal frame that will be used is given by

$$
b=\frac{\tilde{d}}{a(D-2)} .
$$


In this frame, there is no conformal factor multiplying the flat worldvolume directions of the black brane metric. Making this choice has the added advantage that it gives

$$
\Lambda=0 .
$$

Perturbing the equations of motion gives

$$
\begin{aligned}
& \nabla_{N}\left(e^{\alpha \bar{\phi}} \delta F^{N P_{1} \ldots P_{n-1}}\right)+\left(\alpha \partial_{N} \delta \phi\right) e^{\alpha \bar{\phi}} \bar{F}^{N P_{1} \ldots P_{n-1}}-(n-1) e^{\alpha \bar{\phi}} \bar{F}^{N Q\left[P_{2} \ldots P_{n-1}\right.} \nabla_{N} h_{Q}^{\left.P_{1}\right]} \\
&-h_{M}^{N} \nabla_{N}\left(e^{\alpha \bar{\phi}} \bar{F}^{M P_{1} \ldots P_{n-1}}\right)-\nabla_{N}\left(h_{M}^{N}-\frac{1}{2} h \delta_{M}^{N}\right) e^{\alpha \bar{\phi}} \bar{F}^{M P_{1} \ldots P_{n-1}}=0 \\
& \nabla^{2} \delta \phi-h^{M N} \nabla_{M} \nabla_{N} \bar{\phi}+\beta h^{M N} \partial_{M} \bar{\phi} \partial_{N} \bar{\phi}-\partial_{M} \bar{\phi} \nabla_{N}\left(h^{M N}-\frac{1}{2} h \bar{g}^{M N}\right)-2 \beta \partial \bar{\phi} \cdot \partial \delta \phi \\
&+\frac{a}{2(n-1) !} e^{(\alpha+\beta) \bar{\phi}} h^{M N} \bar{F}_{M P_{1} \ldots P_{n-1}} \bar{F}_{N} P_{1} \ldots P_{n-1}-\frac{a}{n !} e^{(\alpha+\beta) \bar{\phi}} \bar{F} \cdot \delta F \\
&-\frac{a}{2 n !} e^{(\alpha+\beta) \bar{\phi}}(\alpha+\beta) \delta \phi \bar{F}^{2}=0 . \\
& \nabla^{2} h_{M N}-\quad \nabla_{M} \nabla^{P}\left(h_{P N}-\frac{1}{2} h \bar{g}_{P N}\right)-\nabla_{N} \nabla^{P}\left(h_{P M}-\frac{1}{2} h \bar{g}_{P M}\right) \\
&-2 R_{P(M} h_{N)}^{M}-2 R_{(M|P Q| N)} h^{P Q}+2 \beta\left(\nabla_{(M} h_{N)}^{P}-\frac{1}{2} \nabla^{P} h_{M N}\right) \partial_{P} \bar{\phi} \\
&-2 \beta \nabla_{M} \nabla_{N} \delta \phi+4\left(k+\beta^{2}\right) \partial_{(M} \bar{\phi} \partial_{N)} \delta \phi \\
&+ \frac{1}{(n-1) !} e^{(\alpha+\beta) \bar{\phi}}\left[2 \delta F_{(M} P_{1} \ldots P_{n-1} \bar{F}_{N) P_{1} \ldots P_{n-1}}-(n-1) h^{P Q} \bar{F}_{M P R_{1} \ldots R_{n-2}} \bar{F}_{N Q} R_{1} \ldots R_{n-2}\right. \\
&+\left.(\alpha+\beta) \delta \phi \bar{F}_{M P_{1} \ldots P_{n-1}} \bar{F}_{N} P_{1} \ldots P_{n-1}\right]=0
\end{aligned}
$$

where bars denote background quantities, and the background metric is used to define covariant derivatives and raise and lower indices.

It is convenient to split the $D$-dimensional indices $M, N$ into indices $i, j$ along the spatial worldvolume directions and indices $\mu, \nu$ in the other directions. Indices $m, n$ will be used to denote directions on the $n$-sphere. The choice of conformal frame made above implies that connection components and curvature components vanish if they have an $i$ index. $z$ will be used to denote spatial worldvolume coordinates and $x$ to denote other coordinates.

\subsection{The Gregory-Laflamme ansatz}

Following Gregory and Laflamme [2], only s-wave perturbations will be considered. For such perturbations,

$$
h^{t m}=h^{r m}=h^{i m}=0, \quad h_{n}^{m}=K(t, r) \delta_{n}^{m} .
$$

Other components, and the dilaton perturbation, are also taken to be independent of the coordinates on the sphere. This implies that the terms involving $h_{M N}$ and $\delta \phi$ in equation 4.10 vanish, just as in [2]. It is therefore consistent to set $\delta F=0$. In [2], it was shown that there was no unstable mode associated with $\delta F$. It seems very likely that the same is true here, so $\delta F=0$ will be assumed from now on. 
GL made the ansatz

$$
h_{M N}=\exp \left(i \mu_{i} z^{i}\right) H_{M N}(x), \quad \delta \phi=\exp \left(i \mu_{i} z^{i}\right) f(x)
$$

and considered only longitudinal perturbations, i.e.,

$$
\begin{aligned}
& H_{i \mu}=i \mu_{i} A_{\mu}(x), \\
& H_{i j}=\mu_{i} \mu_{j} H(x) .
\end{aligned}
$$

The same ansatz will be adopted here. Under gauge transformations, the metric perturbation transforms as

$$
h_{M N} \rightarrow h_{M N}^{\prime}=h_{M N}-\nabla_{M} \xi_{N}-\nabla_{N} \xi_{M},
$$

so under a gauge transformation with $\xi_{M}=\exp \left(i \mu_{i} z^{i}\right) \Xi_{M}$ and $\Xi_{i}=i \mu_{i} \Xi$ the metric perturbation becomes

$$
\begin{aligned}
H_{\mu \nu}^{\prime} & =H_{\mu \nu}-\nabla_{\mu} \Xi_{\nu}-\nabla_{\nu} \Xi_{\mu}, \\
A_{\mu}^{\prime} & =A_{\mu}-\partial_{\mu} \Xi-\Xi_{\mu}, \\
H^{\prime} & =H+2 \Xi .
\end{aligned}
$$

The quantity $H_{\mu \nu}-2 \nabla_{(\mu} A_{\nu)}-\nabla_{\mu} \nabla_{\nu} H$ is gauge invariant under such transformations. Note that it is possible to transform away $A_{\mu}$ and $H$ by a gauge transformation. The following gauge choice can therefore be made

$$
H_{i \mu}=H_{i j}=0 \text {. }
$$

This is not the same gauge as used by Gregory and Laflamme. However, in many respects, it is much simpler to use this gauge that the de Donder gauge that they adopted. The main reason for using this gauge is to relate the classical instability to a negative mode. The negative mode can be viewed as arising from a lower dimensional black hole solution, and is therefore independent of the coordinates $z^{i}$. It therefore appears simplest to adopt a gauge in which the perturbation is independent of these coordinates.

Using the gauge 4.19 and the above ansatz for the form of the perturbation, the dilaton perturbation equation reduces to

$$
\begin{aligned}
-\nabla^{2} f & +2 \beta \partial \bar{\phi} \cdot \partial f+H^{\mu \nu} \nabla_{\mu} \nabla_{\nu} \bar{\phi} \\
& -\beta H^{\mu \nu} \partial_{\mu} \bar{\phi} \partial_{\nu} \bar{\phi}+\partial_{\mu} \bar{\phi} \nabla_{\nu}\left(H^{\mu \nu}-\frac{1}{2} H_{\rho}^{\rho} \bar{g}^{\mu \nu}\right) \\
& -\frac{a}{2(n-1) !} e^{(\alpha+\beta) \bar{\phi}}\left(H^{\mu \nu} \bar{F}_{\mu \rho_{1} \ldots \rho_{n-1}} \bar{F}_{\nu}^{\rho_{1} \ldots \rho_{n-1}}-\frac{\alpha+\beta}{n} \bar{F}^{2} f\right)=\lambda f,
\end{aligned}
$$

wheref $\lambda=-\mu^{2}$. The reason for writing the equations in this form will soon become apparent. The $\mu \nu$ components of the metric perturbation equation are

$$
\begin{aligned}
& -\nabla^{2} H_{\mu \nu}+2 \nabla_{(\mu} \nabla^{\rho} H_{\nu) \rho}-\nabla_{\mu} \nabla_{\nu} H_{\rho}^{\rho}+2 R_{\rho(\mu} H_{\nu)}^{\rho}+2 R_{(\mu|\rho \sigma| \nu)} H^{\rho \sigma} \\
& -\beta\left(2 \nabla_{(\mu} H_{\nu)}^{\rho}-\nabla^{\rho} H_{\mu \nu}\right) \partial_{\rho} \bar{\phi}+2 \beta \nabla_{\mu} \nabla_{\nu} f-4\left(k+\beta^{2}\right) \partial_{(\mu} \bar{\phi} \partial_{\nu)} f \\
& +\frac{1}{(n-1) !} e^{(\alpha+\beta) \bar{\phi}}\left((n-1) H^{\rho \sigma} \bar{F}_{\mu \rho \lambda_{1} \ldots \lambda_{n-2}} \bar{F}_{\nu \sigma}{ }^{\lambda_{1} \ldots \lambda_{n-2}}-\bar{F}_{\mu \lambda_{1} \ldots \lambda_{n-1}} \bar{F}_{\nu} \lambda_{1 \ldots \lambda_{n-1}}(\alpha+\beta) f\right) \\
& =\lambda H_{\mu \nu} .
\end{aligned}
$$

\footnotetext{
${ }^{9}$ Note that $\mu^{2}=\sum_{i} \mu_{i} \mu_{i}$, which should not be confused with the parameter $\mu$ that appears in the black brane solution.
} 
The $\mu i$ and $i j$ components of the metric perturbation equation reduce to

$$
\begin{gathered}
Y_{\mu} \equiv \nabla_{\nu} H_{\mu}^{\nu}-\beta H_{\mu}^{\nu} \partial_{\nu} \bar{\phi}-2 f\left(k+\beta^{2}\right) \partial_{\mu} \bar{\phi}=0, \\
Z \equiv H_{\rho}^{\rho}-2 \beta f=0 .
\end{gathered}
$$

It is straightforward to check that the trace of equation 4.21 together with equations 4.22 and 4.23 implies equation 1.20 . Equation 1.23 can be used to eliminate $f$ from equations 4.21 and 4.22 and then the problem reduces to solving equations 4.21 and 4.22 . Note that equation 4.22 looks rather like a gauge condition. However, this cannot be the case because a gauge has already been chosen, so equation 4.22 should be regarded as an equation of motion.

GL looked for solutions with time dependence $e^{\Omega t}$. That could be done here too, with equation 4.22 used to express two of the four remaining unknown functions $\left(H^{t t}, H^{t r}, H^{r r}\right.$ and $K$ ) in terms of the other two. These could then be substituted into equation 4.21 to give differential equations for these two remaining unknowns. This appears to give a set of equations in far fewer unknowns that those studied in [1, 2]. This difference arises because the gauge choice adopted above is not the same as the one used by GL.

This route will not be pursued here since the aim of this paper is to exhibit a connection between classical instability and Euclidean negative modes. It was argued above that the necessary and sufficient condition for a classical GL instability to exist is that there should exist a static threshold unstable mode. For such a mode,

$$
\Omega=H^{t r}=0 .
$$

\subsection{Boundary conditions}

For a threshold unstable mode, the boundary conditions at the horizon of the black brane are particularly simple. If the background metric is written in the form 3.6 then the perturbed Lorentzian metric can be written

$$
d s^{2}=-U(r)(1+\phi(r, z)) d t^{2}+V(r)^{-1}(1+\psi(r, z)) d r^{2}+R(r)^{2}(1+k(r, z)) d \Omega^{2}+d z^{i} d z^{i}
$$

where $\phi, \psi$ and $k$ are infinitesimal perturbations with $z$-dependence $\exp \left(i \mu_{i} z^{i}\right)$. To examine regularity of this perturbation on the future horizon, one can transform to Eddington-Finkelstein [5] coordinates. One finds that the metric is regular at the horizon if, and only if, the perturbation is bounded as $r \rightarrow r_{+}$and

$$
\phi\left(r_{+}, z\right)=\psi\left(r_{+}, z\right) .
$$

At large $r$, the boundary condition is that the perturbation should decay.

\section{Thermodynamic instability}

In this section, it will be shown that a classical Lorentzian threshold unstable mode exists if, and only if, the black hole obtained by dimensional reduction of the black brane has a Euclidean negative mode. 


\subsection{Wick rotation}

If a threshold unstable mode exists then it can be Wick rotated to Euclidean signature (this will change the sign of $h_{t t}$ ). This gives a solution to the equations describing perturbations around the Euclidean signature black brane solution. As discussed in the introduction for the case of the Schwarzschild black hole, the perturbation that will be related to the Euclidean negative mode will not be the classical perturbation given by $h_{M N}$ and $\delta \phi$ (which are proportional to $\exp \left(i \mu_{i} z^{i}\right)$ ), but rather the (off-shell) perturbation given by the translationally invariant functions $H_{M N}$ and $f$. This perturbation satisfies equations 4.20 and 4.21 , which look rather like eigenvalue equations for small quantum fluctuations around a Euclidean black hole solution. Moreover, since $\lambda=-\mu^{2}$ is negative, it appears that this particular fluctuation has a negative eigenvalue and is therefore a Euclidean negative mode of the black hole. In this section it will be shown that this is indeed the case. This Euclidean perturbation will be referred to as a candidate negative mode.

Since the background solution and the perturbation in question are both translationally invariant, it is often convenient not to distinguish between perturbations of the black brane and those of the black hole obtained by dimensional reduction. A negative mode of the black hole can be regarded as a translationally invariant negative mode of the black brane. In order to avoid having to work in two different conformal frames, the black hole will be studied in the frame in which its metric is given by simply neglecting the flat directions of the black brane metric. Of course, physical quantities such as masses and charges should be calculated in the lower dimensional Einstein frame. However, if a negative mode exists in one conformal frame then one must exist in all other conformal frames since a change of variable in the Euclidean path integral cannot change the number of negative modes.

It is necessary to show that the candidate negative mode is really a physical negative mode. The first thing to check is that it satisfies the appropriate boundary conditions for a quantum fluctuation around the black hole. These are that the fluctuation should decay sufficiently rapidly, and preserve regularity at $r=r_{+}$. The first of these follows from the large $r$ behaviour of the Lorentzian solution, which is clearly not changed by Wick rotation. The second can be seen by Euclideanizing 4.25 (i.e. setting $t=-i \tau)$, dropping the $\exp \left(i \mu_{i} z^{i}\right)$ factors, and then defining a new radial coordinate $\rho$ near $r=r_{+}$by

$$
\rho=\frac{2 \sqrt{r-r_{+}}}{\sqrt{V^{\prime}\left(r_{+}\right)}}\left(1+\frac{1}{2} \psi\left(r_{+}\right)\right) .
$$

In the new radial coordinate, the $\tau \rho$ part of the metric becomes, near $r=r_{+}$,

$$
d \rho^{2}+\rho^{2} \frac{U^{\prime}\left(r_{+}\right) V^{\prime}\left(r_{+}\right)}{4}\left(1-\psi\left(r_{+}\right)+\phi\left(r_{+}\right)\right) d \tau^{2} .
$$

Regularity of the background solutions implies that $\tau \sim \tau+\beta$ and equation 3.7 is satisfied. The perturbation only preserves this regularity if it is bounded and satisfies

$$
\psi\left(r_{+}\right)=\phi\left(r_{+}\right),
$$

and this is clearly satisfied by the Wick rotated mode if it obeys the Lorentzian boundary condition 4.26 . 


\subsection{Negative modes}

The candidate negative mode can be written

$$
\delta g_{\mu \nu}=H_{\mu \nu}, \quad \delta g_{\mu i}=\delta g_{i j}=0, \quad \delta \phi=f .
$$

$H_{\mu \nu}$ and $f$ satisfy the Wick rotated versions of equations 4.20 and 4.21, which can be written as

$$
\mathbf{G}(x) \int d^{D} x^{\prime} \mathbf{O}\left(x, x^{\prime}\right) \mathbf{X}\left(x^{\prime}\right)=\sqrt{g} e^{-\beta \bar{\phi}} \lambda \mathbf{X}(x)
$$

where

$$
\mathbf{X}=\left(\begin{array}{c}
\delta \phi \\
\delta g_{M N}
\end{array}\right), \quad \mathbf{O}\left(x, x^{\prime}\right)=\left(\begin{array}{cc}
\frac{\delta^{2} I}{\delta \phi(x) \delta \phi\left(x^{\prime}\right)} & \frac{\delta^{2} I}{\delta \phi(x) \delta g_{P Q}\left(x^{\prime}\right)} \\
\frac{\delta^{2} I}{\delta g_{M N}(x) \delta \phi\left(x^{\prime}\right)} & \frac{\delta^{2} I}{\delta g_{M N}(x) \delta g_{P Q}\left(x^{\prime}\right)}
\end{array}\right)
$$

and

$$
\mathbf{G}=\left(\begin{array}{cc}
1 & \frac{2 \beta}{D-2} g_{M N} \\
\frac{2 \beta}{D-2} g_{P Q} & {\left[2 g_{M(P} g_{Q) N}-\frac{2}{D-2}\left(1-\frac{2 \beta^{2}}{D-2}\right) g_{M N} g_{P Q}\right]}
\end{array}\right) .
$$

The matrix $\mathbf{G} \equiv G_{I J}$ can be regarded as the metric on the space of fluctuations around a solution of the field equations, i.e., if the fluctuation $\left(\delta g_{M N}, \delta \phi\right)$ is denoted schematically as $\psi_{I}$ then the kinetic term for the fluctuation is $G^{I J} \partial \psi_{I} \cdot \partial \psi_{J}$. The presence of $\mathbf{G}$ in equation 5.5 is required to balance up and down $I, J$ indices.

Equation 5.5 is the equation governing quadratic fluctuations around the Euclideanized black brane solution. So the candidate negative mode satisfies the correct eigenvalue equation and the correct boundary conditions for quantum fluctuations around the black brane, or black hole (by dimensional reduction). The remaining thing to check is that it really is a negative mode, i.e., a fluctuation that decreases the Euclidean action. A mode with eigenvalue $\lambda$ has action

$$
\delta I=\lambda \int d^{D} x \sqrt{\bar{g}} e^{-\beta \bar{\phi}} \mathbf{X}^{2}
$$

where $\mathbf{X}^{2}=\mathbf{X}^{T} \mathbf{G}^{-1} \mathbf{X}=G^{I J} X_{I} X_{J}$. Expanding in terms of $f$ and $H_{\mu \nu}$ gives

$$
\begin{aligned}
\mathbf{X}^{2} & =\left(1-\frac{\beta^{2} D}{D-2}\right) f^{2}+\beta H_{\rho}^{\rho} f+\frac{1}{2} H^{\mu \nu} H_{\mu \nu}-\frac{1}{4}\left(H_{\rho}^{\rho}\right)^{2} \\
& =f^{2}+\frac{1}{2} \tilde{H}^{M N} \tilde{H}_{M N}-\frac{D-2}{4 D}\left(\hat{H}_{M}^{M}\right)^{2}
\end{aligned}
$$

where $\hat{H}_{M N}$ is defined to be the D-dimensional Einstein frame metric perturbation, with $\tilde{H}_{M N}$ its traceless part. It is clear that the metric $\mathbf{G}$ is not positive definite, but has a negative eigenvalue associated with the trace of the Einstein frame metric perturbation. In other words, the kinetic term for this trace has the wrong sign. This is, of course, the conformal factor problem [17].

Equation 5.8 implies that modes for which $\mathrm{X}^{2}<0$ everywhere are negative modes (i.e. decrease the action) if $\lambda>0$. These will be regarded as unphysical because they arise from the conformal factor problem. The modes with $\mathbf{X}^{2}>0$ everywhere are associated with directions in the path integral along which the kinetic terms remain bounded, and therefore if such a mode has $\lambda<0$ then it represents a physical non-conformal negative mode. 
The candidate negative mode was obtained from a Lorentzian solution with $Z=0$, so it has $H_{\rho}^{\rho}=2 \beta f$. This implies

$$
\mathbf{X}^{2}=\left(1-\frac{2 \beta^{2}}{D-2}+\frac{2 \beta^{2}}{n+2}\right) f^{2}+\frac{1}{2} H^{\prime \mu \nu} H_{\mu \nu}^{\prime},
$$

where $H_{\mu \nu}^{\prime}$ is the traceless part of $H_{\mu \nu}$. A sufficient condition for $\mathbf{X}^{2}>0$ is that the coefficient of $f^{2}$ should be non-negative. Using equations 4.3, 4.8 and 2.3, it is easy to show that this occurs if, and only if,

$$
a^{2} \geq \frac{2(D-p-3)^{2}(2-p)}{(D-2)(D-p)} .
$$

This is automatically satisfied for $p \geq 2$. For $p=1$, the cases of interest have to be considered individually. For a $\mathrm{D} p$-brane, $D=10$ and $a=(p-3) / 2$ and it follows that equation 5.11 is satisfied for $p=1$. For the magnetic NS branes considered in [2], one has $D=10$ and $a=(7-p) / 2$, and equation 5.11 is satisfied for $p=1$. Thus, for all the cases of interest, the candidate negative mode is a non-conformal negative mode.

\subsection{Proving the converse}

It has been shown that the classical Lorentzian threshold unstable mode can be converted into a non-conformal Euclidean negative mode by dropping the $\exp \left(i \mu_{i} z^{i}\right)$ factors and Wick rotating. If one accepts that the existence of a non-conformal negative mode implies local thermodynamic instability then it follows that the existence of a Gregory-Laflamme instability implies that there is also a local thermodynamic instability.

To establish equivalence of classical and local thermodynamic stability, it is necessary to prove the converse. As shown above, local thermodynamic instability (negative specific heat) implies the existence of a non-conformal negative mode for the lower dimensional black hole solution, with some eigenvalue $\lambda<0$. This can be oxidized to give a negative mode of the black brane. This will satisfy the Euclidean versions of equations 4.20 and 4.21 so it appears that this can now be Wick rotated and multiplied by $\exp \left(i \mu_{i} z^{i}\right)\left(\right.$ with $\left.\mu^{2}=-\lambda\right)$ to obtain a classical Lorentzian threshold unstable mode.

This conclusion is a little premature. Equations 4.20 and 4.21 are indeed recovered this way, but what about equations 4.22 and 4.23 ? At first sight, it is hard to see how these could emerge from the negative mode since they are associated with the classical equations of motion in the flat extra dimensions, which the negative mode does not "know" about. However, if one takes the trace of equation 4.21 and combines it with equation 4.20 , one can derive

$$
-2 \nabla^{2} Z+2 \beta \partial \bar{\phi} \cdot \partial Z+2 \nabla_{\mu} Y^{\mu}-2 \beta Y \cdot \partial \bar{\phi}=\lambda Z \text {. }
$$

The combination $\nabla^{\nu}-\beta \partial^{\nu} \bar{\phi}$ times equation 4.21 minus $2\left(k+\beta^{2}\right) \partial_{\mu} \bar{\phi}$ times equation 4.20 gives (after a long calculation involving the background equations of motion, the Bianchi identities and spherical symmetry)

$$
\partial_{\mu}\left(-\nabla^{2} Z+\beta \partial Z \cdot \partial \bar{\phi}+\nabla_{\mu} Y^{\mu}-\beta Y \cdot \partial \bar{\phi}\right)=\lambda Y_{\mu}
$$

It follows that if $\lambda \neq 0$ then

$$
Y_{\mu}-\frac{1}{2} \partial_{\mu} Z=0
$$


i.e.,

$$
\nabla_{\nu}\left(H^{\mu \nu}-\frac{1}{2} H_{\rho}^{\rho} \bar{g}^{\mu \nu}\right)=\beta H^{\mu \nu} \partial_{\nu} \bar{\phi}+2\left(k+\beta^{2}\right) f \partial_{\mu} \bar{\phi}+\beta \partial_{\mu} f
$$

This looks very much like a gauge condition on $H_{\mu \nu}$. One is, of course, free to impose a gauge condition on $H_{\mu \nu}$ since this is just the Euclidean metric perturbation for the black hole background, and no gauge condition has yet been imposed on this perturbation. However, it seems rather odd that a gauge condition should emerge from the equations of motion! The resolution of this puzzle is simply that, for $\lambda \neq 0$, the above equations are not equations of motion, and in fact the right hand side of the eigenvalue equation is not gauge invariant. A similar phenomenon would occur in Maxwell theory: the equation $\partial_{\nu} \delta F^{\mu \nu}=\lambda \delta A^{\mu}$ implies $\partial \cdot \delta A=0$ when $\lambda \neq 0$. Therefore, this eigenvalue equation is only valid Lorentz gauge. In a different gauge, the right hand side of the eigenvalue equation would be different.

Further progress can be made by using equation 5.14 to eliminate $Y_{\mu}$ from equation 5.12, giving

$$
-\nabla^{2} Z+\beta \partial Z \cdot \partial \bar{\phi}=\lambda Z
$$

which implies

$$
\int d^{D} x \sqrt{\bar{g}} e^{-\beta \bar{\phi}}(\partial Z)^{2}=\lambda \int d^{D} x \sqrt{\bar{g}} e^{-\beta \bar{\phi}} Z^{2}
$$

The left hand side of this equation is positive. A normalizable negative mode would give a negative right hand side, so it follows that such a mode must have $Z=0$. Using equation 5.14 it follows that a normalizable negative mode must have $Z=Y_{\mu}=0$, so equations 4.22 and 4.23 are indeed satisfied. This completes the proof that that the threshold classical Lorentzian unstable mode in the gauge 4.19 is obtained from the negative mode in the gauge 5.15 simply by Wick rotation and multiplying by $\exp \left(i \mu_{i} z^{i}\right)$ with $\mu^{2}=-\lambda$.

\section{Conclusions}

It has been explained why there should be a relationship between classical and thermodynamic stability of black branes, as conjectured by Gubser and Mitra. Various assumptions were made which means that the above discussion falls well short of a rigorous proof. It is convenient to summarize the arguments used above, to emphasize where these assumptions were made.

A) Local thermodynamic stability of a magnetically charged black brane is equivalent to positivity of the specific heat of the black hole obtained by dimensional reduction. If the black brane is locally thermodynamically unstable then so is the black hole, so it will have a negative mode. It was assumed that this is an s-wave, but this is probably acceptable since it seems very likely that higher partial waves would have higher eigenvalues. This negative mode can be oxidized to give a negative mode of the black brane. If a suitable gauge is chosen then this negative mode can be converted into a classical threshold unstable mode as described above. Such a mode would be expected to separate stable short wavelength fluctuations of the black brane from unstable long wavelength fluctuations, so the existence of such a mode indicates that a classical Gregory-Laflamme instability exists. So local thermodynamic instability implies classical instability.

B) For the converse, if a black brane is classically unstable then the instability is expected to occur only at long wavelengths. Therefore there should exist a threshold unstable mode that 
is independent of time. Provided this mode is of the longitudinal s-wave form discussed by Gregory and Laflamme, then it is possible to choose a gauge in which the components of the metric perturbation along the spatial worldvolume directions vanish. Dropping the $\exp \left(i \mu_{i} z^{i}\right)$ factors and Wick rotating then gives an s-wave negative mode of the black hole. This implies the existence of a local thermodynamic instability of the black hole, assuming that the results of [36] extend to the black holes considered here. It then follows that the black brane must also be locally thermodynamically unstable.

The assumptions involved in proving A) that local thermodynamic instability implies classical instability seem rather mild. The assumptions involved in proving the converse B) are less obvious, so it would be interesting to see if these could be given better justification. To see why proving B) rigorously is hard, consider the case when the black brane has positive specific heat. Proving B) in this case amounts to proving that the black brane is classically stable, which involves considering all fluctuations around the black brane, not just those of the GL form.

If a black brane is sufficiently far from extremality then it should behave much like an uncharged black brane and therefore have negative specific heat. It then follows from argument A) above that the black brane will be classically unstable. As the brane approaches extremality, argument A) establishes that the brane will be classically unstable as long as the specific heat remains negative. So argument A) extends the results of GL to any magnetically charged black brane with negative specific heat. If argument B) is correct then the instability should vanish when the specific heat becomes positive.

It has been explained that the reason GL found an instability that persisted all the way down to extremality is that the branes they studied always have negative specific heat. Had they chosen to work with branes whose specific heat became positive near extremality then, according to B), they would not have found an instability near extremality.

For a black brane of a type II supergravity theory, with the exception of the D3-brane, it is always possible to dualize the field strength so that the brane is magnetically charged and therefore falls within the class of solutions considered in this paper. The non-extremal NS5, D5 and D6 branes always have negative specific heat so A) implies that they are always classically unstable. However, the F1, D1, D2 and D4-branes have a specific heat that becomes positive near extremality. A) establishes that they should be classically unstable when their specific heat is negative, and B) implies that they should become classically stable near extremality. Note that the F-string and D-string have the same stability properties, as do the NS5 and D5-branes, as one would expect from the $S L(2, R)$ symmetry of IIB supergravity. The major omission from this paper is, of course, the black D3-brane. I think that it is likely that the above analysis could be extended to cover this case, as well as the other dyonic black brane solutions given in [26]. If this is the case then the black D3-brane should be stable near extremality.

The M2 and M5-branes are not covered by the analysis above but one can infer their stability properties from the behaviour of the F-string and D4-branes, which are obtained by dimensional reduction on a worldvolume direction. If the higher dimensional brane were classically unstable to a perturbation of the form considered above then one could reduce in a direction orthogonal to $\mu_{i}$ and the instability would persist in the lower dimensional solution. Since a GL instability is not expected for the near-extremal F-string or D4-brane, this implies that the near extremal M2 and M5-branes must be classically stable, in agreement with the specific heat analysis.

The GM conjecture is supposed to cover any black brane with a non-compact translational symmetry. The branes considered in this paper were all assumed to have flat worldvolumes. 
However, it might be possible to extend the analysis to branes with Ricci flat spatial worldvolume metrics of the "Kaluza-Klein" form

$$
d s^{2}=e^{\alpha \phi}(d \tau+A)^{2}+e^{\beta \phi} \gamma_{i j} d y^{i} d y^{j}
$$

where $A$ is a 1 -form independent of $\tau$, the coordinate along the non-compact direction. The classical instability would presumably involve fluctuations proportional to $\exp (i \mu \tau)$.

Perhaps the most striking result of this paper is that it is possible to show that many black branes are classically unstable without resorting to the arduous numerical analysis of [1, 2]. Instead one just has to calculate the specific heat. So a simple thermodynamic calculation replaces a difficult classical calculation. This can be contrasted with black hole thermodynamics, where the temperature and entropy are, in principle, obtained by a long calculation 《4, but in practice are determined by simple classical quantities, namely the surface gravity and horizon area. Understanding these connections between classical and quantum properties of black holes remains one of the most interesting problems in quantum gravity.

\section{Acknowledgements}

I would like to thank Fay Dowker, Steven Gratton, Ruth Gregory, Stephen Hawking, Tim Prestidge and Simon Ross for illuminating discussions, Fay Dowker and Ruth Gregory for comments on a draft of this paper, Roberto Emparan for comments on the first preprint version, and Tim Prestidge for allowing me to use unpublished results. This work was funded by PPARC.

\section{References}

[1] R. Gregory and R. Laflamme, Phys. Rev. Lett. 70, 2837 (1993).

[2] R. Gregory and R. Laflamme, Nucl. Phys. B428, 399 (1994).

[3] J.M. Bardeen, B. Carter and S.W. Hawking, Commun. Math. Phys. 31, 161 (1973).

[4] S.W. Hawking, Commun. Math. Phys 43, 199 (1975).

[5] S.W. Hawking and G.F.R. Ellis, The large scale structure of space-time, Cambridge University Press (1973).

[6] S.S. Gubser and I. Mitra, hep-th/0009126.

[7] S.S. Gubser and I. Mitra, hep-th/0011127.

[8] J. Maldacena, Adv. Theor. Math. Phys. 2, 231 (1998); hep-th/9711200.

[9] E. Witten, Adv. Theor. Math. Phys. 2, 505 (1998); hep-th/9803131.

[10] C. Csaki, H. Ooguri, Y. Oz and J. Terning, JHEP 01, 017 (1999); hep-th/9806021.

[11] R. Gregory and R. Laflamme, Phys. Rev. D51, R305 (1995); hep-th/9410050. 
[12] S.S. Gubser, Nucl. Phys. B551, 667 (1999); hep-th/9810225.

[13] C.V. Vishveshwara, Phys. Rev. D1, 2870 (1970).

[14] M.J. Duff and J.T. Liu, Nucl. Phys. B554, 237 (1999); hep-th/9901149.

[15] G.W. Gibbons and S.W. Hawking, Phys. Rev. D15, 2752 (1977).

[16] S.W. Hawking in General Relativity, an Einstein Centenary Survey, eds S.W. Hawking and W. Israel (Cambridge University Press, 1979).

[17] G.W. Gibbons, S.W. Hawking and M.J. Perry, Nucl. Phys. B138, 141 (1978).

[18] S.W. Hawking, Phys. Rev. D13, 191 (1976).

[19] D.N. Page, Phys. Rev. D18, 2733 (1978).

[20] D.J. Gross, M.J. Perry and L.G. Yaffe, Phys. Rev. D25, 330 (1982).

[21] T. Prestidge, unpublished.

[22] R. Gregory and R. Laflamme, Phys. Rev. D37, 305 (1988).

[23] G.T. Horowitz and A. Strominger, Nucl. Phys. B360, 197 (1991).

[24] R. Güven, Phys. Lett. B276, 49 (1992).

[25] M.J. Duff, J.X. Lu, Nucl. Phys. B416, 301 (1994); hep-th/9306052.

[26] M.J. Duff, H. Lu and C.N. Pope, Phys. Lett. B382, 73 (1996); hep-th/9604052.

[27] G.W. Gibbons and K. Maeda, Nucl. Phys. B298, 741 (1988).

[28] J.X. Lu, Phys. Lett. B319, 29 (1993); hep-th/9304159.

[29] J.W. York, Jr., Phys. Rev. D33, 2092 (1986).

[30] T. Prestidge, Phys. Rev. D61, 084002 (2000); hep-th/9907163.

[31] B. Whiting and J.W. York, Jr., Phys. Rev. Lett. 61, 1336 (1988).

[32] S.W. Hawking and G.T. Horowitz, Class. Quant. Grav. 13, 1487 (1996); gr-qc/9501014.

[33] R.B. Mann and S.F. Ross, Phys. Rev. D52, 2254 (1995); gr-qc/9504015.

[34] S.W. Hawking and S.F. Ross, Phys. Rev. D52, 5865 (1995); hep-th/9504019.

[35] L.D. Landau and E.M. Lifshitz, Statistical Physics, 3rd edition part 1, Pergamon, Oxford (1980).

[36] T. Prestidge, Ph.D. thesis, University of Cambridge (2000). 